American Journal of Applied Sciences 6 (4): 565-575, 2009

ISSN 1546-9239

(C) 2009 Science Publications

\title{
A Study of Fatigue Data Editing using the Short-Time Fourier Transform (STFT)
}

\author{
S. Abdullah, C.K.E. Nizwan and M.Z. Nuawi \\ Department of Mechanical and Materials Engineering, Universiti Kebangsaan Malaysia, \\ 43600 Bangi, Selangor, Malaysia
}

\begin{abstract}
This study presents the development of the STFT-based fatigue data editing technique that will be used as a tool to accelerate for accelerating fatigue testing. This technique was performed by removing low amplitude cycles contained in the original signal in order to produce a shortened signal using the Short-Time Fourier Transform (STFT) parameter. The effectiveness of STFT power spectrum was validated using an SAE random fatigue data in order to indicate the relationship between STFT parameter and fatigue damage. The data was separated into two segments, i.e., damage and nondamage segments based on the $100 \%$ retention of the original fatigue damage. For the editing process, the STFT power spectrum distribution was used as the parameter to identify the damaged segment according to the power spectrum Cut-Off Level (COL). The low amplitude cycles with power spectrum lower than COL value were then removed from the original signal. Thus, a new edited signal was obtained which has retained almost $100 \%$ of the original fatigue damage and has equivalent signal statistic. The edited signal was found to be approximately $84 \%$ of the time duration of the original signal.
\end{abstract}

Key word: Fatigue data editing, random loading, signal processing, STFT

\section{INTRODUCTION}

Fatigue durability testing of the mechanical structure is performed extensively in all industries as one of the parts in design process. In the real application, the fatigue loading services such as stresses on a car wheel, bending moment on the stub axle of a car, stresses on the rear axle of a passenger car etc. are variable amplitude histories ${ }^{[1]}$. The histories often contain a large percentage of small amplitude cycles and the fatigue damage for these cycles can be small. Therefore, in many cases the fatigue loading history were edited by removing those small amplitudes cycles in order to produce representative and meaningful yet economical testing ${ }^{[2]}$.

For fatigue life assessment study, fatigue data editing is described as a method of summarising the fatigue data by removing small amplitude cycles for reducing the testing time and cost. Two key factors were suggested in order to achieve efficient design and modification processes to ensure adequate fatigue performance, i.e., the fatigue damage should be as accurate as possible and the component durability tests should be as short as possible ${ }^{[3]}$. Several fatigue data editing techniques have been developed for use in the time domain analysis ${ }^{[4]}$. Some of the previous algorithms were developed for eliminating low amplitude cycles, hence to retain high amplitude cycles $^{[4-6]}$. In the frequency domain, a time history is low pass filtered on the basis that high frequency cycles have small amplitudes which are not damaging ${ }^{[6]}$. The filtering method does not shorten the signal because it does not provide the time base information.

The time-frequency approach has been applied to the problem of fatigue data editing through the compromise between the time- and frequency-based views of a signal. The Short-Time Fourier Transform (STFT) or windowed Fourier transform is one of the methods for transforming the time domain signal into the time-frequency domain. In addition, the STFT adapted the Fourier transform to analyse only a small section of the signal at one specific time ${ }^{[7]}$. Finally, STFT provides information on when and at what frequencies a signal occurs.

This study focuses on two parts, i.e., STFT validation with an SAE data and editing process. For the STFT validation, the fatigue data editing was applied in time domain analysis to shorten the original signal with $100 \%$ damage retains. The editing process was performed using the GlyphWorks ${ }^{\circledR}$ software package, which sliced the strain history range less than gate value. Gate value was obtained from strain

Corresponding Author: S. Abdullah, Department of Mechanical and Materials Engineering, Universiti Kebangsaan Malaysia, 43600 Bangi, Selangor Malaysia Tel: +60-3-89216518 Fax: +60-3-89259659 
amplitudes and mean stress evaluation at cut-off point or fatigue limit of the particular material. The nondamage and damaged segment in time history was recorded. The STFT method was used to transform the time domain fatigue signal into time-frequency domain. The spectrogram of STFT will be transposed into the time history distribution to be compared with the time history non-damage and damage segment in order to study the effectiveness between STFT spectrogram and fatigue damage.

For the editing process, the STFT method was used to transform the time domain signal into the timefrequency domain in order to trace the low power level contained in the original signal. Those parts were then removed from the original signal in order to generate a new edited signal which has segments of high amplitude cycles. For this reason, low amplitude cycles which have been removed have minimal or no fatigue damage potential. Therefore, the original fatigue damage can be retained in the edited signal produced at the end of the process. Finally, for this study, the effectiveness of this technique was validated based on the fatigue damage retention in the shortened signal and it was then compared to the original signal.

\section{MATERIALS AND METHODS}

For many automotive components, the primary mode of failure can be attributed to fatigue damage resulting from the application of variable amplitude loading. Predicting the life of a part stressed above the endurance limit is at best a rough procedure ${ }^{[8]}$ especially for components like the automobile engine, steering and suspension parts ${ }^{[9]}$. For these cases, the strain-based approach is commonly used to predict fatigue life ${ }^{[10]}$. The strain-life fatigue model relates the plastic deformation that occurs at a localized region where fatigue cracks begin to the durability of the structure. This model is often used for ductile materials at relatively short fatigue lives. This approach can also be used where there is little plasticity at long fatigue lives. Therefore, this is a comprehensive approach that can be used in place of the stress-based approach.

Current industrial practice for fatigue life prediction is to use the Palmgren-Miner (PM) linear damage rule ${ }^{[10]}$. For strain-based fatigue life prediction, this rule is normally applied with strain-life fatigue damage models, such as the Coffin-Manson relationship ${ }^{[11,12]}$, i.e.,

$$
\varepsilon_{\mathrm{a}}=\frac{\sigma_{\mathrm{f}}^{\prime}}{\mathrm{E}}\left(2 \mathrm{~N}_{\mathrm{f}}\right)^{\mathrm{b}}+\varepsilon_{\mathrm{f}}^{\prime}\left(2 \mathrm{~N}_{\mathrm{f}}\right)^{\mathrm{c}}
$$

where $\mathrm{E}$ is the material modulus of elasticity, $\varepsilon_{\mathrm{a}}$ is a true strain amplitude, $2 \mathrm{~N}_{\mathrm{f}}$ is the number of reversals to failure, $\sigma_{\mathrm{f}}$ is a fatigue strength coefficient, $\mathrm{b}$ is a fatigue strength exponent, $\varepsilon_{\mathrm{f}}$ is a fatigue ductility coefficient and $\mathrm{c}$ is a fatigue ductility exponent.

In order to estimate a fatigue damage event where mean stress/strain is involved, functional solutions, in addition to the normal fatigue stress/strain life curves, are introduced to predict mean stress and mean strain effects. Experimental results show that mean strain gives a second-order effect if no severe work hardening has taken place ${ }^{[13]}$. Consequently, the damage parameters are usually developed to consider mean stress effects on fatigue behaviour. Different mean stress effects have been documented for a variety of materials and testing techniques, with the result that various mean stress theories for strain-life relationship have emerged and no consensus exists that any one of them is superior to the others ${ }^{[13]}$. For examples, the Morrow $^{[14]}$ approach seems to work reasonably well for steels while the Smith-Watson-Topper $(\mathrm{SWT})^{[15]}$ appears to give good results for a wide range of materials and is a good choice for general use ${ }^{[13]}$. The Morrow's strain-life model is mathematically defined as the following expression:

$$
\varepsilon_{\mathrm{a}}=\frac{\sigma_{\mathrm{f}}^{\prime}}{E}\left(1-\frac{\sigma_{\mathrm{m}}}{\sigma_{\mathrm{f}}{ }^{\prime}}\right)\left(2 \mathrm{~N}_{\mathrm{f}}\right)^{\mathrm{b}}+\varepsilon_{\mathrm{f}}^{\prime}\left(2 \mathrm{~N}_{\mathrm{f}}\right)^{\mathrm{c}}
$$

and the SWT strain-life model is defined according to this formulation:

$$
\sigma_{\max } \varepsilon_{a}=\frac{\left(\sigma_{f}^{\prime}\right)^{2}}{E}\left(2 N_{f}\right)^{2 b}+\sigma_{f}^{\prime} \varepsilon_{f}^{\prime}\left(2 N_{f}\right)^{c}
$$

where $\sigma_{\mathrm{m}}$ is the mean stress and $\sigma_{\max }$ is the maximum stress, applicable for both Eq. 2 and 3.

From the strain-life model approach, the number of reversals or $2 \mathrm{~N}_{\mathrm{f}}$, were determined in order to find cumulative fatigue damage. The values of $\sigma_{\mathrm{f}}{ }_{\mathrm{f}}, \mathrm{E}, \varepsilon_{\mathrm{f}}, \mathrm{b}$ and $\mathrm{c}$ were the material properties of the particular material, while the value of $\varepsilon^{\prime}{ }_{a}$ can be obtained from the rain flow cycle counting method. The cumulative fatigue damage was then determined using the Palmgren-Miner (PM) linear damage rule.

Fourier analysis is a one of the method to analyse random data based on the frequency domain analysis. The frequency analysis data is typically presented in graphical form as Power Spectral Density (PSD). Essentially a PSD display the amplitude of each sinusoidal wave of a particular frequency. Frequency is given on the $\mathrm{x}$-axis. The mean squared amplitude of a 
sinusoidal wave at any frequency can be determined by finding the area under the PSD over that frequency range. Unfortunately, Fourier transform analysis has a drawback as it does not provide the time localization information. Therefore time-frequency domain analysis was applied to solve that problem. Many timefrequency analyses are based on windowed or shorttime Fourier transforms ${ }^{[16]}$. Sliding data windows were used to obtain time-localised spectra which together put up the time-frequency representation of the data.

The STFT is performed by dividing the signal into small sequential or overlapping data frames, for which the Fast Fourier Transform (FFT) was applied to each data frame ${ }^{[17]}$. The output of successive STFT can provide a time-frequency representation of the signal. In order to accomplish this, the signal is truncated into short data frames by multiplying it by a window so that the modified signal is zero outside the data frame. In order to analyse the whole signal, the window is then translated into a time and reapplied to the signal. The STFT is composed by the local spectra of segments of the primary function, as viewed through a translating window of fixed shape. The local spectra at all points on the primary time axis constitute the STFT ${ }^{[18]}$. The STFT is generally expressed as the following:
$\operatorname{STFT}(\mathrm{t}, \mathrm{f})=\int_{-\infty}^{\infty} \mathrm{h}(\mathrm{t}) \mathrm{w}(\mathrm{t}-\tau) \exp (-2 \pi \mathrm{if} \tau) \mathrm{d} \tau$

where $\mathrm{h}$ is the primary function, $\tau$ is the time and $\mathrm{f}$ is the frequency. The position of the translating window $w$ is determined by $t$, which has the same units as $\tau$. If $w$ is replaced with the value of 1 in Eq. 2, the STFT reduces to $\mathrm{H}$, i.e., the Fourier transform of $h$. The modulus of the STFT is also known as the spectrogram.

In the related study ${ }^{[19]}$, the STFT spectrogram has been applied in fatigue analysis for detection and monitoring of hidden fatigue crack growth. The STFT method was also widely used in locating the structure defect especially for gear and cutting tool using vibration data due to its capability to detect highly vibration event ${ }^{[20,21]}$. In this study, the STFT method was used to transform the fatigue signal into timefrequency representation in order to detect the damaging event contained in the signal. STFT measures the energy in a time-frequency neighbourhood, specified by a resolution box. The damaging event will be determined based-on level of energy.

\section{RESULTS}

The validation process STFT-based fatigue data editing was presented as a flowchart in Fig. 1. In order

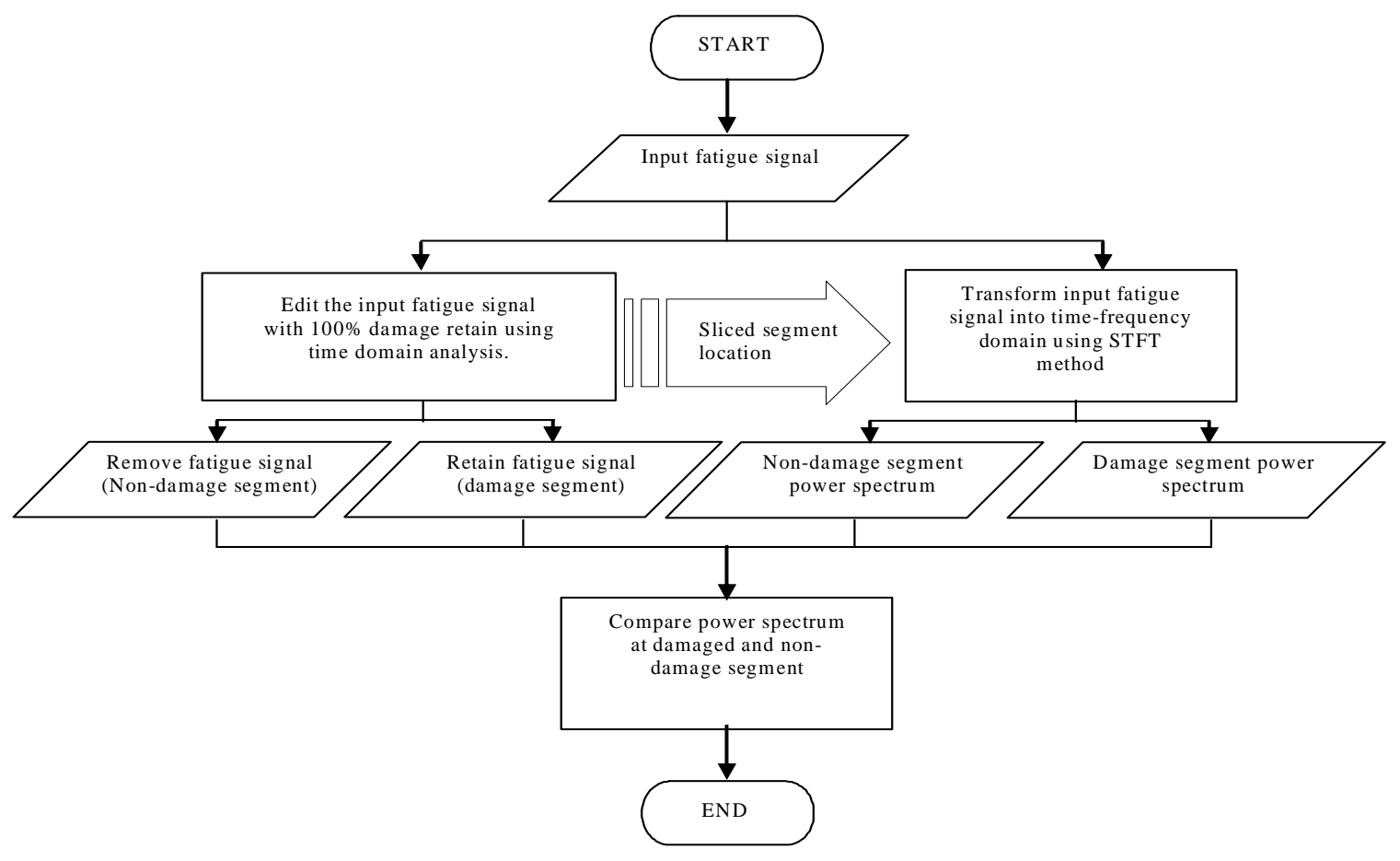

Fig. 1: Simplified flowchart for the validation of STFT effectiveness 


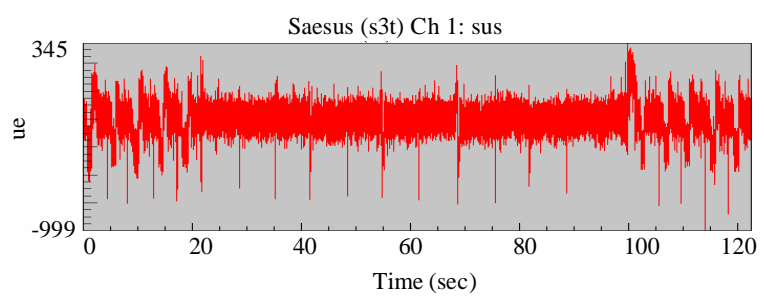

Fig. 2: Time history plot of the SAESUS fatigue signal

to achieve this purpose, the strain time histories named as SAESUS (this data was obtain from the database of Society of Automotive Engineers or SAE) was used for evaluating the newly developed fatigue data editing technique associated with the STFT. The data was collected on a suspension component of a car and it was assumed to be sampled at $204.8 \mathrm{~Hz}$ for 25,000 data points. It gave the total record length of the signal of $122 \mathrm{sec}$, as illustrated in Fig. 2. The signal was chosen due to the prior success with it by another researcher ${ }^{[22]}$ in the fatigue history editing using wavelet approach.

The time domain fatigue signal was then edited for shortening the signal length with the removal of low amplitude cycles. These cycles were removed based on time domain analysis. The module for fatigue damage editing in the GlyphWorks ${ }^{\circledR}$ software package was applied to perform the time signal shortening. The editing process was retained at $100 \%$ damage level of the original signal. In this process all the strain range less than the gate value were removed, for which the gate value was calculated based on Coffin-Manson strain-life relationship. The selected material for the simulation purpose was the SAE1045 steel and this type of material was commonly used in the automotive industry ${ }^{[23]}$ for fabricating a lower suspension arm. The material properties and their definitions are given in Table 1. For the SAE 1045 steel material, the gate value is the strain range that gave the fatigue life value of $2 \times 10^{8}$ reversals.

The removed low strain range cycle from the original signal was shortening the length of the signal. The $100 \%$ retained damage strain range edited was successful removed approximately 50\% low amplitude cycles with 57 seconds time reduced, as illustrated in Fig. 3. For this case, the removed data was assumed as non-damage segments because the departures of those segments did not change the value of the total fatigue damage. In Fig. 3a, the filled areas represent the segments which contribute to the minimal or no damage potential.

For the STFT analysis, the time history signal was separated into a number of windows using the Gaussian window with 128 of window size. The Gaussian
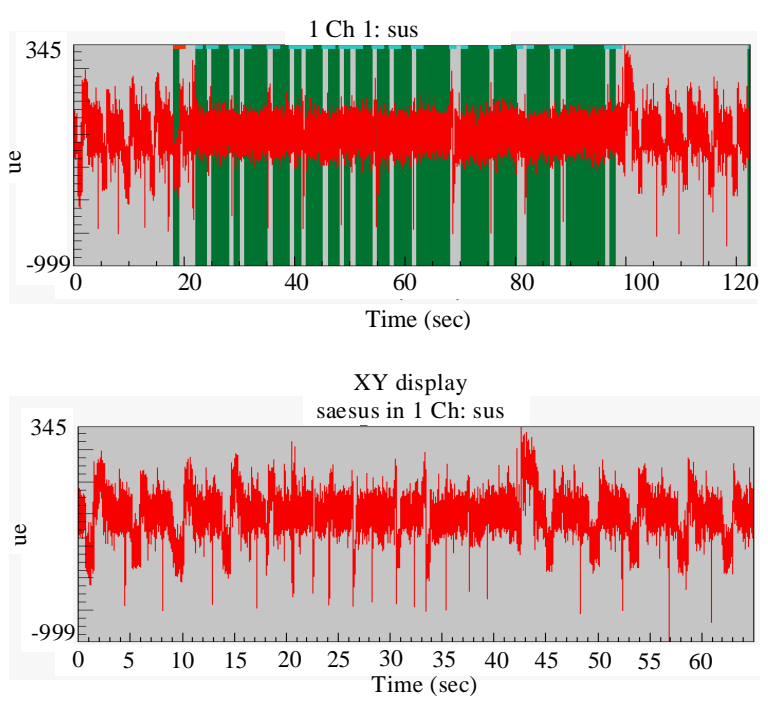

Fig. 3: The analysis of SAESUS: (a) The 122-second original time history (b) The 65-second edited time history

Table 1: Mechanical properties of the SAE1045 steel

\begin{tabular}{ll}
\hline Properties & SAE1045 steel \\
\hline Ultimate tensile strength, $\mathrm{S}_{\mathrm{u}}(\mathrm{MPa})$ & 621 \\
Modulus of elasticity, $\mathrm{E}(\mathrm{GPa})$ & 204 \\
Fatigue strength coefficient, $\sigma_{\mathrm{f}}(\mathrm{MPa})$ & 948 \\
Fatigue strength exponent, $\mathrm{b}$ & -0.092 \\
Fatigue ductility exponent, c & -0.445 \\
Fatigue ductility coefficient, $\varepsilon_{\mathrm{f}}$ & 0.26 \\
\hline
\end{tabular}

window was used since one simultaneously achieves an optimal time and frequency resolution ${ }^{[24]}$. The 120 number of overlaps were used in order to provide the high resolution in the time representation. For each window, the Fourier transform was applied for the calculation of the power spectral level contained in each window. The power distribution was gained using the Power Spectral Density (PSD) that produced the spectrogram of the STFT. For this case, the PSD is defined as the power distribution of the signal and represent at $\mu \varepsilon^{2} \mathrm{~Hz}^{-1}$ as the unit. The STFT plot of the original fatigue signal showed a two-dimensional view of the power distribution, as observed in the timefrequency plane. This result was plotted in Fig. 4, showing the different colour contours, i.e., the red colour for the highest energy content and followed by yellow, green, blue and white.

According to the spectrogram parameter obtained in the STFT processing, the power spectrum of the fatigue signal was decomposed into a time domain data in order to represent the time history power spectrum distribution. The magnitudes of time domain power spectrum were obtained from the accumulative power 


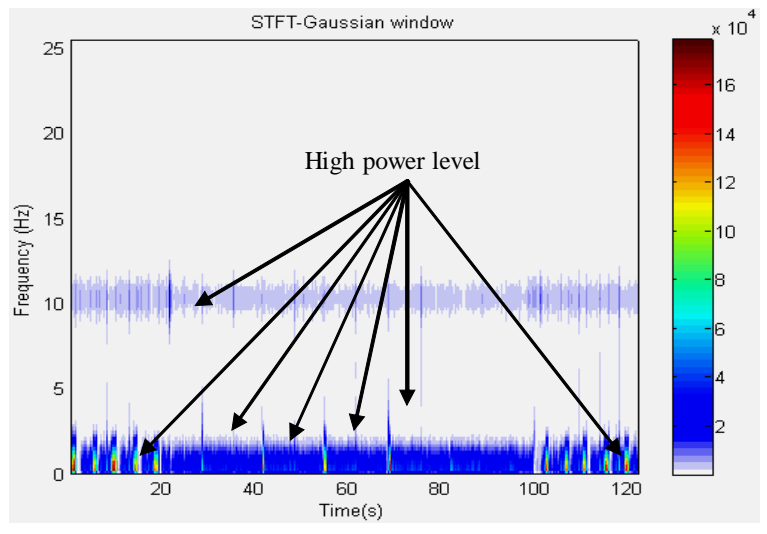

Fig. 4: The time-frequency localisation of the SAE fatigue signal based on the STFT approach
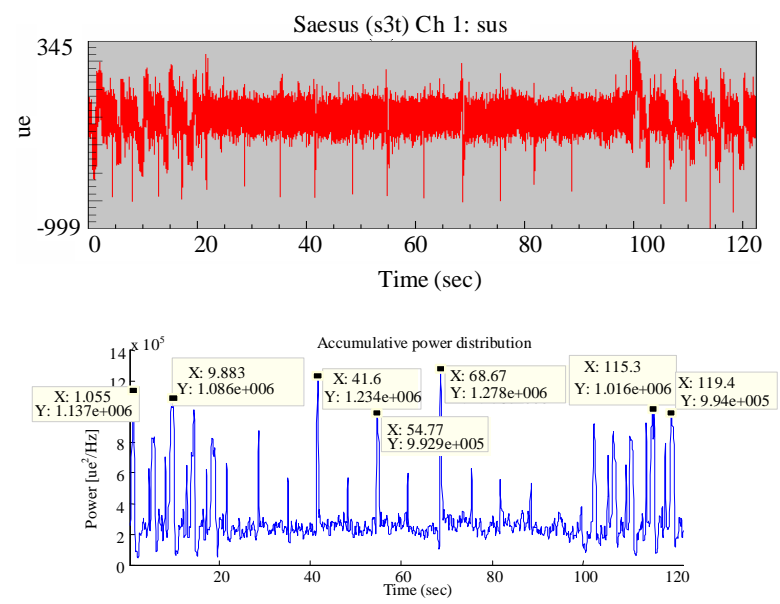

Fig. 5: (a) SAESUS strain time history, (b) The power spectrum in time history representation

distribution along the frequency band for each time interval. Thus, it's provided the power level information at time location. The STFT power spectrum distribution is illustrated in Fig. 5. The figure shows the equality between magnitudes of power spectrum and strain magnitude especially at 1, 10, 42, 55, 69, 115 and $120 \mathrm{sec}$ time locations, where both of the power spectrum and strain possess high magnitudes.

From the simulation of the fatigue history editing at $100 \%$ damage retained, the output signal was separated into two new signals, i.e., the damage signal and the non-damage signal. The damage signal is the signal that contained the cycles associated to the fatigue damage. On the other hand, the non-damage signal contained the cycles which were not damaging. For the validation purposes, the power spectrum of each signal was also investigated in order to study the efficiency of

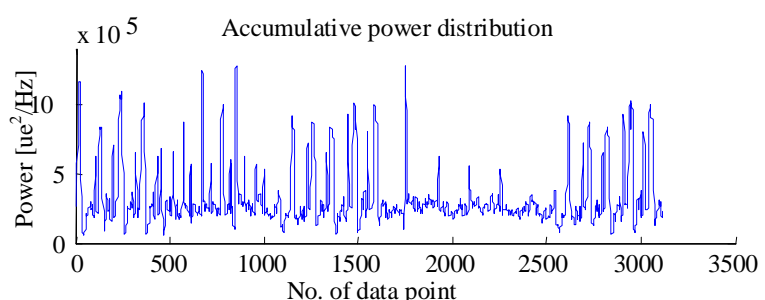

(a)

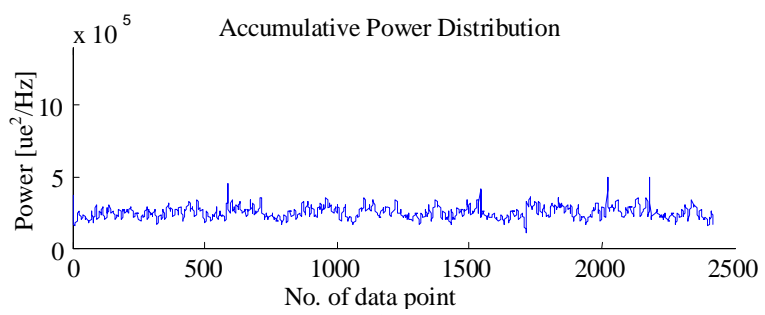

(b)

Fig. 6: Power spectrum at: (a) the damage segment (b) the non-damage segment

STFT parameter based on fatigue damage event. The power spectrum of each signal is shown in Fig. 6, for which Fig. 6a shows that the power spectrum of the non-damage signal that consist higher power spectrum level. The non-damage signal produced the lower power spectrum level at the minimum value, as shown in Fig. 6b, which most of the power spectrum values were below $4 \times 10^{5} \mu \varepsilon^{2} \mathrm{~Hz}^{-1}$. The findings showed that the power spectrum gained from the STFT method was enable us to detect the damage event of the fatigue signal, as higher power spectrum presents the damage part.

The flowchart in Fig. 7 shows the process of fatigue data editing using the STFT method. The overall process consists of time-frequency transformation, time history power spectrum transposition and elimination of the low amplitudes cycles. The output of successive STFT can provide a time-frequency representation of the signal.

Based on the spectrogram parameter obtained in the STFT processing, the power of the fatigue signal was decomposed into a time domain data in order to represent the power distribution in the time history. The power spectrum display in the time domain provided the time location containing the low power spectrum cycle. Accordingly, the low energy cycles will be eliminated for summarising the signal length without compromising the original fatigue damage potential. For a specific fatigue data, low energy cycles mean these cycles had a low amplitude strain which is not damaging. 


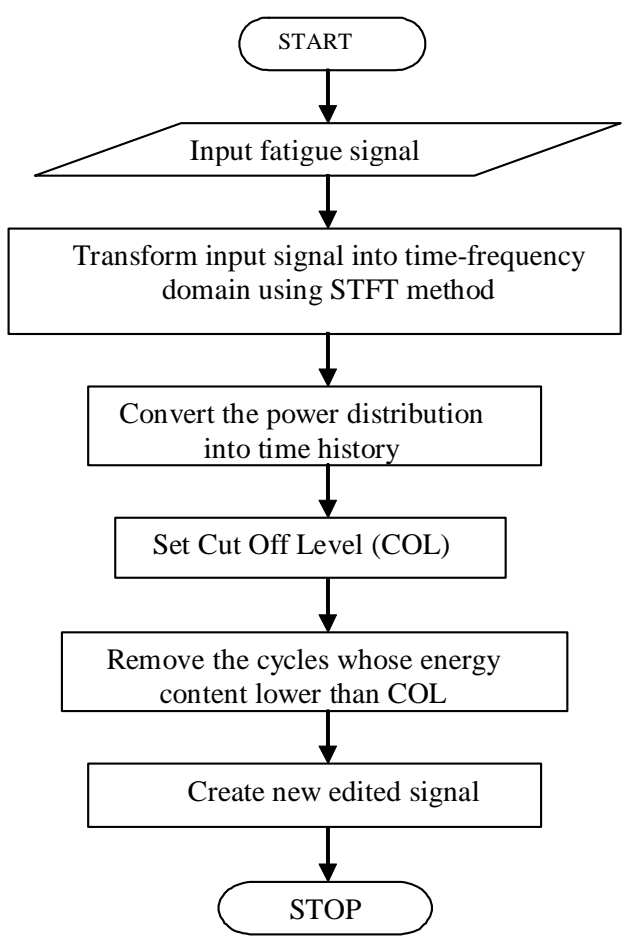

Fig. 7: The flowchart for fatigue data editing using STFT-based computational algorithm

In order to solve the subject matters of this paper, a STFT-based computational algorithm was developed in order to analyse the signal according to the fatigue damage calculation and also to remove cycles with low energy content. Thus, the cycles with higher energy content has been retained for further analysis. These cycles were then jointed to produce the new edited signal, which has shorter time length. In addition, this edited signal should also have equivalent fatigue damage to the original signal.

The low energy cycles were removed from the time domain signal based on the location of low energy cycle in time history power spectrum distribution. A new parameter called Cut-Off Level (COL), which represents the minimum power value to be retained from the original signal was set. This means that, the cycles with power spectrum level below than COL value will be eliminated. Thus, a new shortened edited signal was generated which neglected low amplitude cycles.

For the validation purposes, the fatigue damage potential for both original and edited signals were calculated in order to study the efficiency of the edited signal based on the fatigue damage retention. The fatigue damage was calculated based on strain life model which applied the Coffin-Manson strain-life

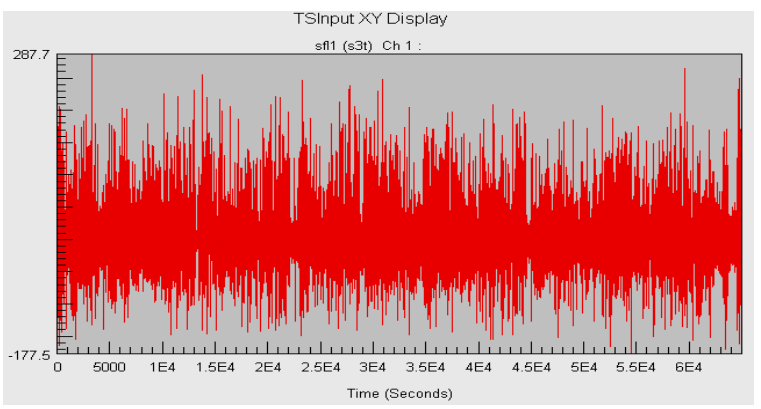

(a)

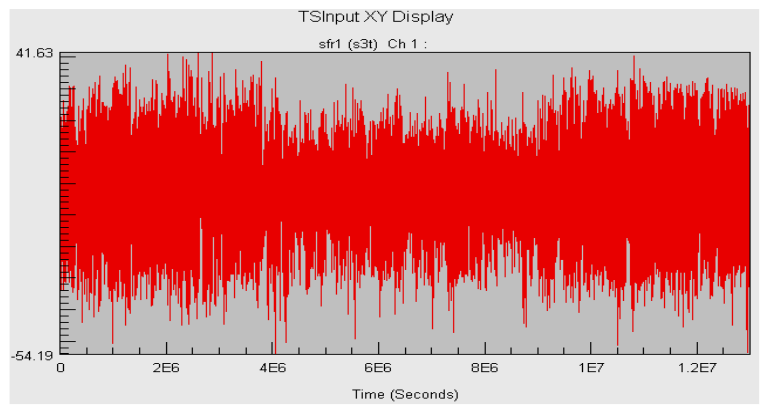

(b)

Fig. 8: Time history signal of that was measured on the different side of lower suspension arm of a car: (a) left lower suspension arm, (b) right lower suspension arm

relationship. In this study, the optimum edited signal was determined based on the shortest signal with the minimal fatigue damage deviation when compared to the original signal and that retains the original signal behaviour.

Two input signals were used to observe the efficiency of the newly developed fatigue data editing algorithm using the STFT approach. Both signals were measured on the left and right lower arm suspension of a car that was travelling on a country road. It was sampled at $200 \mathrm{~Hz}$ for $12,000,000$ data points. The time history plots for the signals were shown in Fig. 8 .

For the analysis purposes, two segments from the overall signal were selected which contained of 30,000 data point for each segment. Those signals were selected due to the highest fatigue damage content. The fatigue damage for each segment was plotted in Fig. 9. The first selected signal, named as D1 (Fig. 10a) was measured on the front left lower suspension arm that contained the highest fatigue damage segment and the second signal, D2 (Fig. 10b), was simultaneously measured with the D1 signal on the front right lower suspension arm.

Using the STFT-based newly developed computational algorithm, the time history D1 and D2 


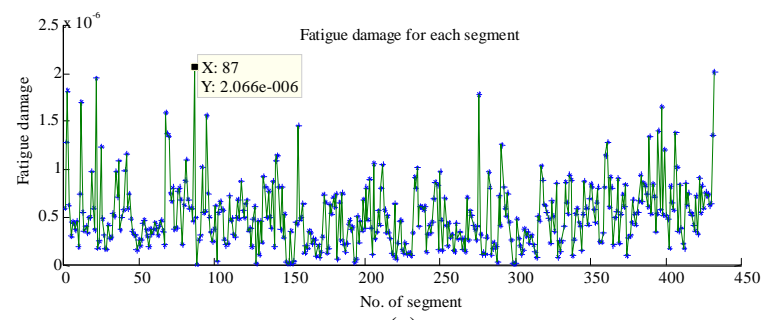

(a)

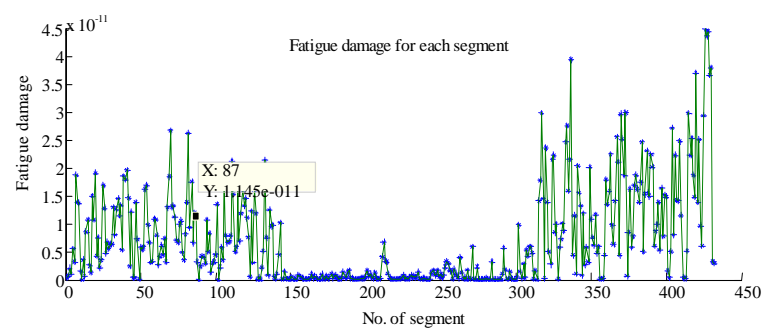

(b)

Fig. 9: Fatigue damage distribution calculated based on 30000 data points for each segment: (a) signal measured on the left lower suspension arm, (b) signal measured on the right lower suspension arm

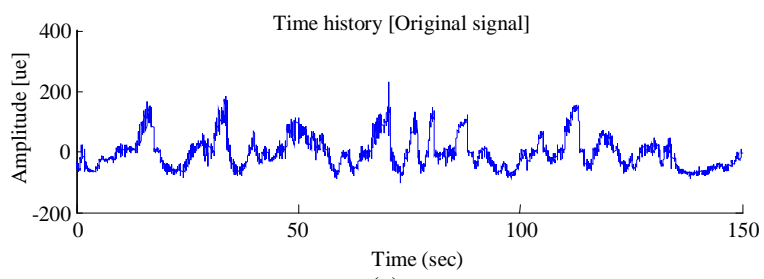

(a)

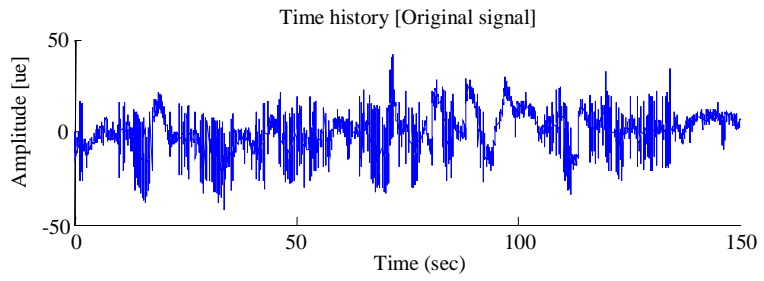

(b)

Fig. 10: Time history signal of: (a) The D1 signal (b) The D2 signal

signal was then transformed into time-frequency representation. Those signals were separated into a number of windows using the Gaussian window with 64 of window size. The 60 number of overlaps were used in order to provide the high resolution in the time representation where higher time resolution provided better time information for further analysis. The timefrequency power spectrum for both signals was plotted

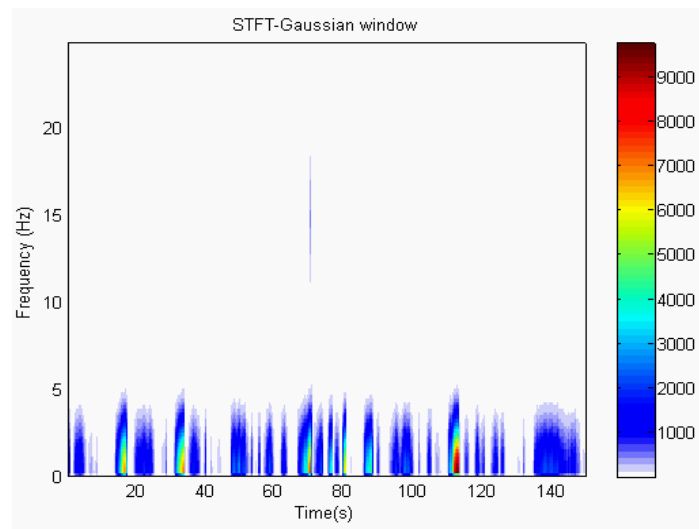

(a)

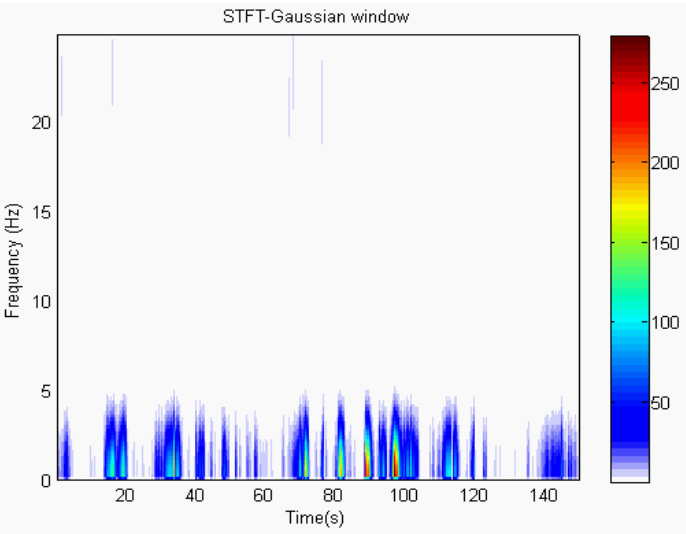

(b)

Fig. 11: The STFT localisation for: (a) D1 signal, (b) D2 signal

in Fig. 11. As can be seen from this figure, higher magnitude distribution was observed with low frequencies and lower magnitude distribution was observed with high frequencies. The time-frequency power spectrum distribution was then converted into time representation by accumulating the power spectrum at each time scale. Thus, a set of power spectrum at particular time was gained. The power spectrum in time history for D1 and D2 signals were plotted in Fig. 12. As the STFT power spectrum has a significant relationship with the fatigue damage potential distribution, the STFT spectrogram can be utilized as the parameter for this fatigue data editing.

For the editing process, the power spectrum level was used as the parameter to set the gate value (Cut-Off Level or also known as COL) for eliminating process. The eliminating process was carried out by removing the low amplitude cycles which contain the power spectrum lower than COL value. Various COL values were used in order to exhibit the effectiveness of the 


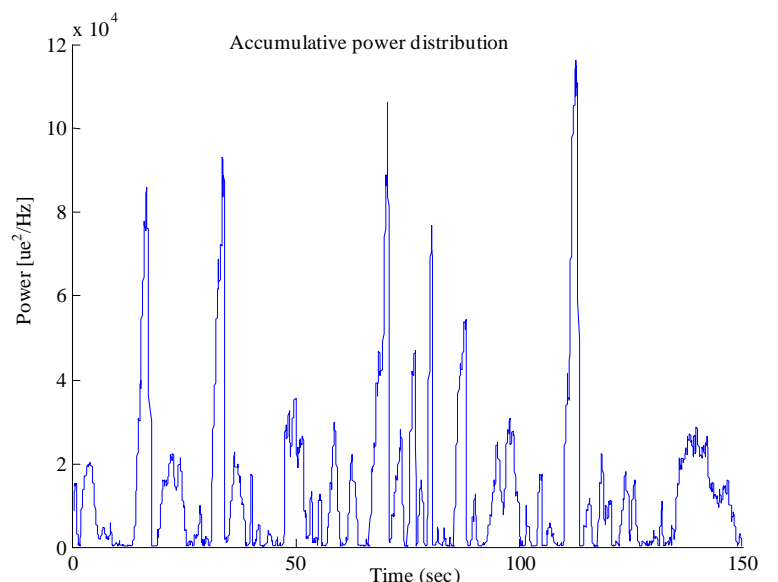

(a)

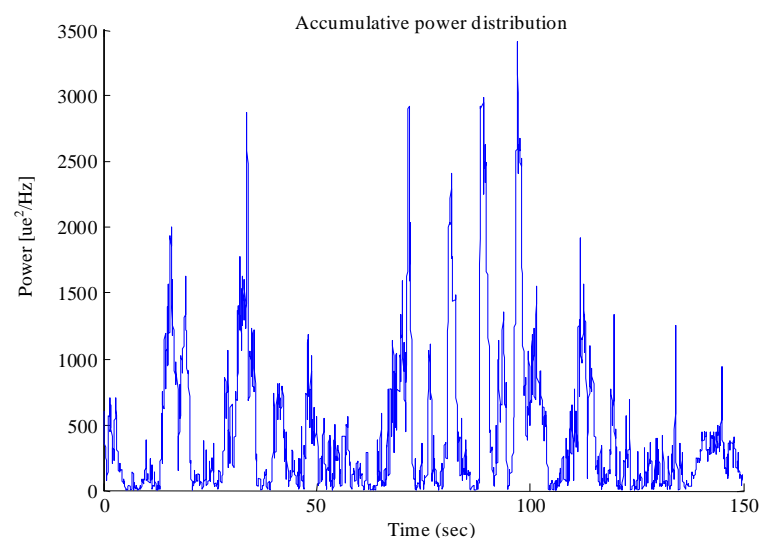

(b)

Fig. 12: Power spectrum in the time history representation for: (a) D1 signal, (d) D2 signal

edited signal with respect to the fatigue damage retention. The editing process at a particular COL value produced a new edited signal. For each edited signal, the fatigue damage was determined and compared with the original signal in order to obtain the optimum edited signal. The fatigue damage was estimated by utilizing the Coffin-Manson, Morrow and SWT strain-life models contained in the GlyphWorks ${ }^{\circledR}$ software.

The simulation process provided the fatigue damage distribution for each cycles as shown in Fig. 13. The fatigue damage of the signal was the cumulative of the fatigue damage for each cycles contained in that signal. The fatigue damage values for each edited signal were plotted in Fig. 14, showing the changes of fatigue damage against the COL values for all strain-life fatigue damage models. The fatigue damage shows decrement when COL values were ascending because of the departure of more low amplitude cycles. In order to retain the originality of the
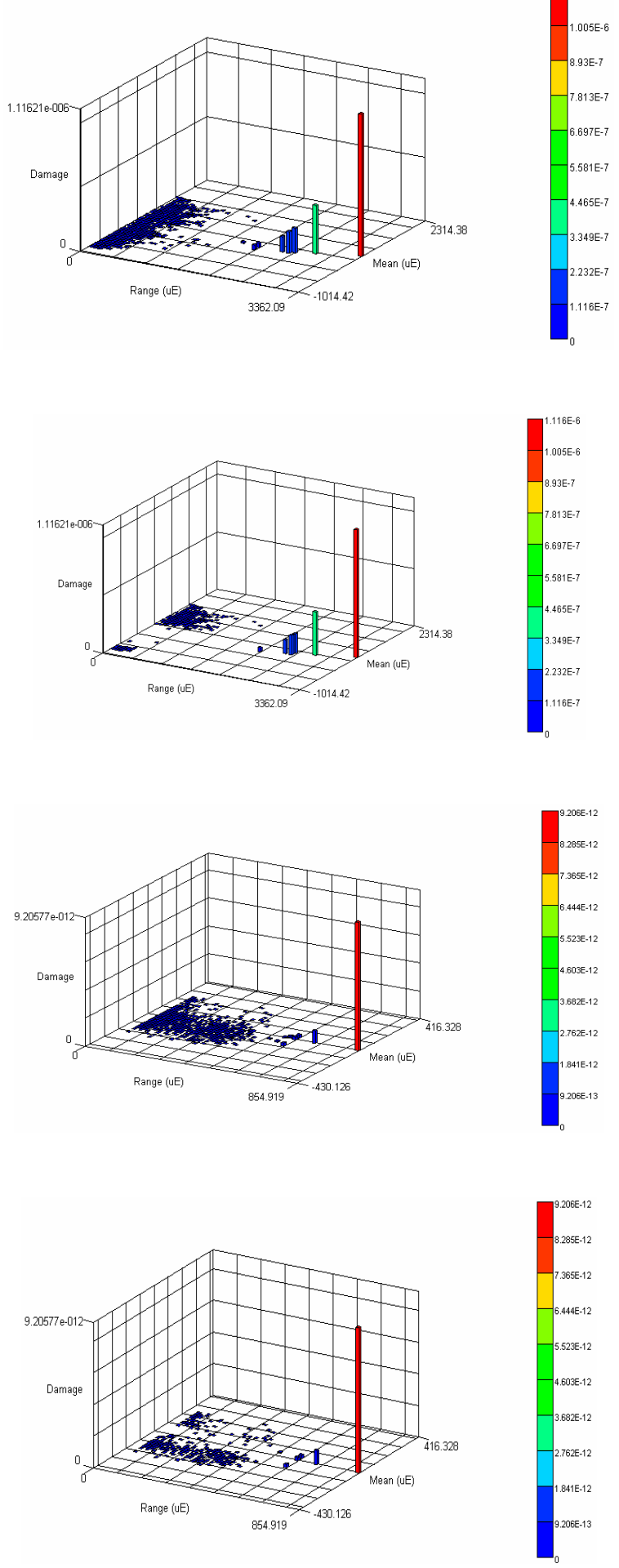

Fig. 13: The distribution of the fatigue damage potential for: (a) D1 signal, (b) D1 edited signal, (c) D2 signal, (d) D2 edited signal 
Am. J. Applied Sci., 6 (4): 565-575, 2009

Table 2: The compression characteristics between the original and edited signals

\begin{tabular}{|c|c|c|c|c|c|c|c|}
\hline \multirow[b]{2}{*}{ Data } & \multirow[b]{2}{*}{ Signal length(s) } & \multirow[b]{2}{*}{ Mean } & \multirow[b]{2}{*}{ RMS } & \multirow[b]{2}{*}{ Kurtosis } & \multicolumn{3}{|l|}{ Fatigue damage } \\
\hline & & & & & Coffin-Manson & Morrow & SWT \\
\hline $\begin{array}{l}\text { Original D1 } \\
\text {. }\end{array}$ & 150 & -2.09 & 51.30 & 3.30 & $2.07 \times 10^{-6}$ & $2.38 \times 10^{-6}$ & $3.25 \times 10^{-6}$ \\
\hline Edited D1 & 126 & -2.43 & 54.00 & 3.00 & $2.07 \times 10^{-6}$ & $2.38 \times 10^{-6}$ & $3.25 \times 10^{-6}$ \\
\hline Original D2 & 150 & 0.20 & 9.10 & 3.53 & $1.147 \times 10^{-11}$ & $1.12 \times 10^{-11}$ & $1.07 \times 10^{-11}$ \\
\hline Edited D2 & 127 & 0.22 & 9.43 & 3.30 & $1.147 \times 10^{-11}$ & $1.12 \times 10^{-11}$ & $1.07 \times 10^{-11}$ \\
\hline
\end{tabular}

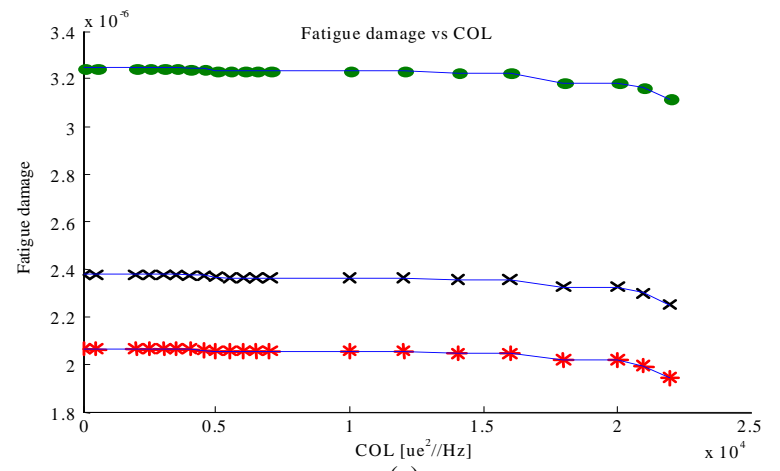

(a)

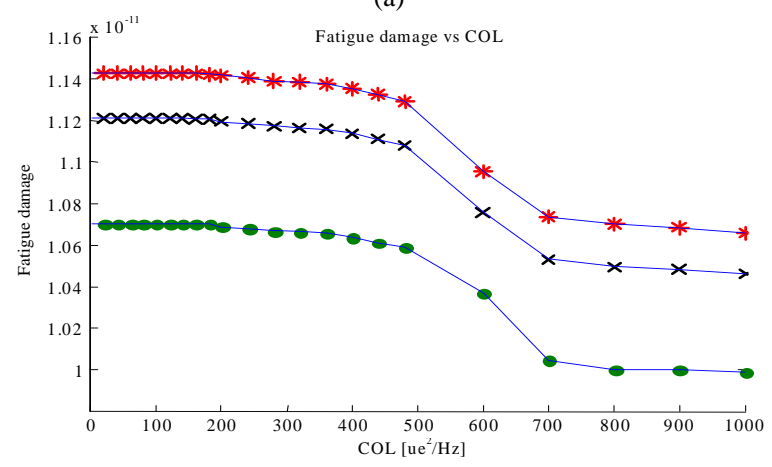

(b)

Fig. 14: The fatigue damage changes over the COL values for: (a) D1 signal, (b) D2 signal

signal, the statistical parameter of the edited signal should be equivalent to the original signal. For this case, the $10 \%$ difference in the root-mean-square and kurtosis values between the edited and the original signals was used for analysing experimental road load data sets. This is important in order to retain the signal energy and amplitude ranges ${ }^{[25,26]}$.

From the result, the optimum COL value for D1 was 500 and $20 \mu \varepsilon^{2} \mathrm{~Hz}^{-1}$ for D2. Both of the edited signals gained from those COL value were retained in the majority of the fatigue damage and were approximately same as the original signal and they also retained the statistical parameters with below than $10 \%$ deviation. Figure 15 represent the edited signal for both D1 and D2 signals. The D1 edited signal recorded 126 seconds signal length which reduced $16 \%$ of the original signal length, while the D2 edited signal was

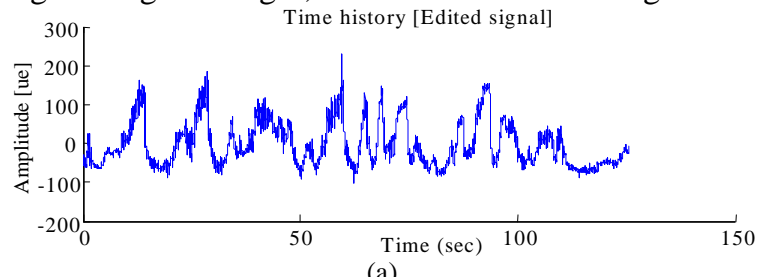

(a)

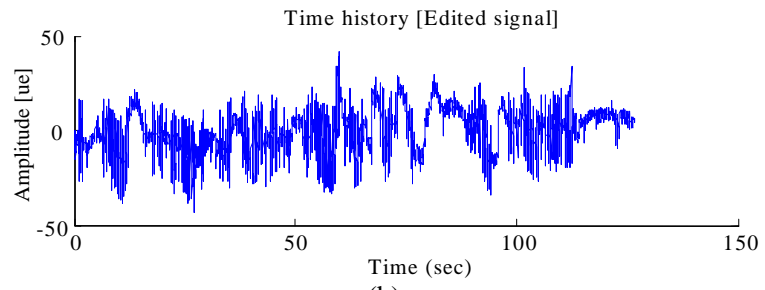

(b)

Fig. 15: The time history plots for: (a) the D1 edited signal, (b) the D2 edited signal

$84.67 \%(127 \mathrm{sec})$ of the original signal. The compression characteristics between the original and edited signals were shown in Table 2.

In overall, the analysis findings of this paper suggested that the STFT-based fatigue data editing can successfully remove the low amplitude cycles with respect to the power spectrum distribution, that retain higher fatigue damage segments in the time history. With the basis of the statistical parameter retention between the original and the edited signals, this technique produced the highly accurate edited signal which was similar to the original signal. The STFT power spectrum shows relatively adequate with damage event in the fatigue signal and is a very useful tool for damage detection in the fatigue signal. The extraction of damaging events successfully created a new edited signal which retained the majority of the fatigue damage.

\section{CONCLUSION}

This study discussed the study of a fatigue data editing technique in time-frequency domain by using STFT method. The STFT-based computational 
algorithm was developed to remove the low amplitude cycles which were contained in the original signal. The validation of the effectiveness of STFT was done by using the SAE data, called SAESUS. From the result, the damage segment contained high power spectrum and the non-damage segments were located in the lower part of the power spectrum. Obviously, the non-damage power spectrum has the power level below than $4 \times 10^{5}$ $\mu \varepsilon^{2} \mathrm{~Hz}^{-1}$ which is close to the minimum value. It was shown that the power spectrum gained from the STFT algorithm has a significant relationship with the fatigue damage distribution.

The editing process was performed based on the COL parameter which eliminated the cycle that contain power spectrum lower than COL value. In the presented case study, two new shortened edited signals, i.e., the D1 edited signal and the D2 edited signal were obtained. The edited signals gave conspicuous decreases of the signal length. The D1 edited signal had $126 \mathrm{sec}$ of the time length, with the shortening of $16 \%$ of the original signal length. Similarly, the D2 edited signal was only $127 \mathrm{sec}$ of the time length, which is about $15.3 \%$ reduction from the original signal length. Both of the signals also retained the major signal statistics with below than $10 \%$ of the root-mean-square value (represents the vibration signal energy in a time series) and the kurtosis value (represent the amplitude range in a time series).

In terms of the applicability of the shortened signal, this kind of signal can be normally used in the durability laboratory scale fatigue test. Such test is very important in the fatigue design criteria, especially for the task of accelerated fatigue testing. Finally, this method is suggested as an alternative technique in fatigue durability study, especially for the automotive engineering field.

\section{ACKNOWLEDGEMENT}

The authors would like to express their gratitude to Universiti Kebangsaan Malaysia and Ministry of Science, Technology and Innovation, through the fund of 03-01-02-SF0052, for supporting these research activities.

\section{REFERENCE}

1. Sonsino, C.M., 2006. Fatigue testing under variable amplitude loading. Int. J. Fatigue, 2006 (www.sciencedirect.com).
2. Stephens, R.I., P.M. Dindinger and J.E. Gunger, 1997. Fatigue damage editing for accelerated durability testing using strain range and SWT parameter criteria. Int. J. Fatigue, 19: 599-606.

3. Duquesnay, D.L., 2002. Application of overload data to fatigue analysis and testing. Appl. Automat. Tech. Fatigue Fracture Test. Anal., 1411: 165-180.

4. El-Ratal, W., M. Bennebach, X. Lin and R. Plaskitt, 2002. Fatigue life modelling and accelerated test for components under variable amplitude loads. Symposium on Fatigue Testing and Analysis under Variable Amplitude Loading Conditions, Tours, France.

5. Conle, A., G. Grenier, H. Johnson, S. Kemp, G. Kopp and M. Morton, 1997. Service History Determination. SAE Fatigue Design Handbook AE-22. Warrendale USA: Society Automotive Engineers Inc, pp: 115-144.

6. Austen, I. and R. Gregory, 1995. Component test during duration prediction and accelerated by fatigue analysis and fatigue editing. VTT Symposium, 3: 169-87.

7. Matlab User's Guide, 2005. Matlab r2006a, The Math Works.

8. Juvinal, R.C. and K.M. Marshek, 2000. Fundamentals of Machine Component Design. 3rd Edn,, John Wiley and Sons, USA.

9. Conle, A. and R. Landgraf, 1983. A fatigue analysis program for ground vehicle components. Proceedings of the International Conference on Digital Techniques in Fatigue, London, pp: 1-28.

10. Dowling, N.E., 1999. Mechanical Behaviour of Materials: Engineering Methods for Deformation Fracture and Fatigue. 2nd Edn., Prentice Hall, New Jersey.

11. Coffin, L.F., 1954. A study of the effect of cyclic thermal stresses on a ductile metal. Trans. ASME, 79: 931-950.

12. Manson, S.S., 1965. Fatigue: A complex subjectsome simple approximation. Exp. Mech., 5: 193226.

13. Bannantine, J.A., J.J. Comer and J.L. Handrock, 1990. Fundamentals of Metal Fatigue Analysis. Prentice-Hall, New Jersey USA.

14. Morrow, J.D., 1968. Fatigue Properties of Metal Fatigue Design Handbook. Society of Automotive Engineers.

15. Smith, K.N., P. Watson and T.H. Topper, 1970. A stress-strain function for the fatigue of metals. J. Mater., 5: 767-778.

16. Gabor, D., 1946. Theory of communication, J. Inst. Elect. Eng., 93: 429-457. 
17. Kiymik, M.K., I. Guler, A. Dizibuyuk and M. Akin, 2005. Comparison of STFT and wavelet transform methods in determining epileptic seizure activity in EEG signal for real-time application. Comput. Biol. Med., 35: 603-616.

18. Pinnegar, C.R. and L. Mansinha, 2003. A method of time-time analysis: The TT-Transform. Digital Signal Process., 13: 588-603.

19. Ihn, J.B. and F.K .Chang, 2004. Detection and monitoring of hidden fatigue crack growth using a built-in piezolectric sensor/actuator network: I. Diagnostic. Inst. Phys. Publi., Smart Mater. Struct. 13: 609-620.

20. Sung, C.K., H.M. Tai and C.W. Chen, 2000. Locating defects of a gear system by the technique of wavelet transform. Mechanism Machine Theory, 35: 1169-1182.

21. Kima, B.S., S.H. Lee, M.G. Lee, J. Nib, J.Y. Song and C.W. Lee, 2007. A comparative study on damage detection in speed-up and coast-down process of grinding spindle-typed rotor-bearing system. J. Mater. Process. Tech., 187-188: 30-36.
22. Oh, C.S., 2001, Application of wavelet transform in fatigue history editing. Int. J. Fatigue, 23: 241250 .

23. Khalil, M. and T.H. Topper, 2003. Prediction of crack-opening stress levels for 1045 as-received steel under service loading spectral. Int. J. Fatigue, 25: 149-157.

24. Shimmel, M. and J. Gallart, 2005. The Inverse STransform in filters with time-frequency localization. IEEE Trans. Signal Process., 53: 4417-4422.

25. Giacomin, J., A. Steinwolf and W.J. Staszewski, 2001. Application of Mildly Nonstationary Mission Synthesis (MNMS) to automotive road data. ATA 7th International Conference on the New Role of Experimentation in the Modern Automotive Product Development Process, May, 23-25.. Florence, Italy.

26. Abdullah, S., J.A. Giacomin and J.R. Yates, 2004. A mission synthesis algorithm for fatigue damage analysis. Proc. Inst. Mech. Eng., Part D, J. Automobile Eng., 218: 243-258. 